\title{
Nutritional knowledge in an Italian population of children, pre-adolescents and adolescents
}

\author{
Anna Tallarini ${ }^{1}$, Alessandra Zabeo ${ }^{2}$ and Anita Ferraretto ${ }^{2, *}$ \\ 'Via G. Leopardi 16, 24069 Trescore Balneario, Bergamo, Italy: ${ }^{2}$ Dipartimento di Scienze Biomediche per la \\ Salute, Università degli Studi di Milano, LITA, Via Fratelli Cervi 93, 20090 Segrate, Milan, Italy
}

Submitted 4 May 2012: Final revision received 11 January 2013: Accepted 22 January 2013: First published online 27 February 2013

\begin{abstract}
Objective: To evaluate general knowledge about nutrition in an Italian population of children, pre-adolescents and adolescents.

Design: Knowledge about nutrition-related items such as healthy eating, breakfast, snacks, fast food, beverages, fruits and vegetables, cereals and tubers, meat/ fish/legumes/eggs, milk and dairy products, fats and dressings, and sweets was analysed by means of a self-administered questionnaire (QuesCA IT) containing thirty-one questions, that was translated and adapted from a Swiss version (QuesCA) previously used in Geneva and Vaud.

Setting: North of Italy (Bergamo, Milan).

Subjects: Students ( $n$ 614) belonging to two different age groups: 9-11 years (GR1) and 12-16 years (GR2).

Results: Data analysis showed that nutritional knowledge varied in relation to the age of the participants, increasing in particular in the older group, although this difference was not statistically significant for all the considered items. Nutritional knowledge also varied in relation to the gender of the participants, with females in particular seeming to possess better cognition. For each age group there was poor knowledge about the items healthy diet, snacks, milk and dairy products, meat/fish/ legumes/eggs, and fats and dressings. Moreover, the percentage of participants who declared own knowledge as insufficient was higher in GR2 compared with GR1. Conclusions: The present research demonstrates a lack of knowledge about the main concepts of healthy nutrition both in the youngest and oldest participants of the survey. This evidence, together with the presence of higher self-consciousness in GR2, should be taken into account in specific educational interventions during the school period.
\end{abstract}

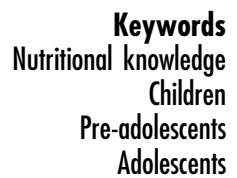

In the last decades the fight to overcome obesity and its associated pathologies among the youngest in Western populations has become urgent. In more countries governments have begun specific actions directed to teach youngsters about a healthy lifestyle in which nutrition accounts for a key role. In this context the first step is to understand youngsters' real level of knowledge about nutrition, although the positive relationship between nutritional knowledge and behaviour ${ }^{(1,2)}$ in some cases has not resulted to be statistically significant ${ }^{(3,4)}$. A possible explanation for this controversy can be found in: (i) the methodologies employed, which sometimes are not completely adequate for this kind of search; (ii) the statistical analysis; and (iii) the usage of investigations designed for a specific field, such as for lipids ${ }^{(1,3,5-10)}$, and thus not fully appropriate for different kinds of investigation. Nutritional knowledge is not therefore a guarantee of a subsequent coherent action, but it is important that people, besides being free to act, are conscious of their choices.
However, this issue remains of interest and a valid tool to determine nutritional knowledge is needed when an action towards nutritional education must be taken, since to know exactly the lack of information can help to plan direct and precise interventions to solve the problem.

Among the different methodologies, Contento et al. ${ }^{(4)}$ identify the nutrition questionnaire as the best performing test to obtain information not only about nutrition-related knowledge, but also about school and life habits.

On this basis, the aim of the present study was to investigate, by means of a self-administered questionnaire, the real knowledge about food and nutrients of Italian children, pre-adolescents and adolescents belonging to two different age ranges, 9-11 years (GR1) and 12-16 years (GR2), in order to understand at which level it is better to plan to inform the youngest population how to eat safely.

An Italian version (QuesCA IT) of the QuesCA, a validated and published questionnaire developed by Kruseman et $a l^{(11)}$, was used. The QuesCA started from the 
individuation of the major themes of interest in nutrition and it was validated and used with success. In particular, the QuesCA presents the following features: (i) it investigates practical knowledge and not just theoretical knowledge; (ii) the choice of items is accurate; (iii) it has a clear layout; (iv) it asks the population in the survey to define some concepts such as healthy nutrition by means of true/false answers and also to indicate the more balanced meal among a group, a difficult but very important question, to understand the level of knowledge; and (v) the age group analysed is of interest because includes children, pre-adolescents and adolescents.

The QuesCA IT is entirely similar to the original and includes thirty-one questions about the major aspects of nutrition, i.e. macronutrients, sweets and beverages, and others more inherent to the concepts of a healthy diet, such as breakfast, fast food and snacks, finishing with a self-assessment about nutritional knowledge. The QuesCA IT was distributed in the schools of Milan and Bergamo, two of the most populated and cosmopolitan towns in northern Italy, and thus representative of the north industrialized part of Italy.

Results obtained represent a useful snapshot of the concepts about nutrition and health among north Italian children, pre-adolescents and adolescents, and therefore could be considered a useful starting point for educational campaigns.

\section{Experimental methods}

\section{Participants}

Six hundred and twenty-three students coming from three Italian schools in the towns of Bergamo and Milan were recruited to participate to the project. The three schools recruited for the surveys were chosen due the possibility for students to attend the primary and secondary school in the same building. The total participants belonged to Primary, Secondary and Further Education (sixteen classrooms) in Milan and to Primary and Secondary Education (fourteen classrooms) in Bergamo.

The students surveyed were divided in two groups: aged 9-11 years (GR1) and aged 12-16 years (GR2). The choice of these two age ranges is based on the observation that children aged 9-11 years are more influenced by their family customs, while pre-adolescents and adolescents in the age range 12-16 years are more influenced by the media, their experiences shared on the World Wide Web and prefer to find information and help outside the family. Moreover, the present subdivision reflects intellectual maturity and the general level of knowledge of the groups is very similar to the one adopted in the work of Kruseman et $a l .{ }^{(11)}$ for which the questionnaire was formulated and validated.

Before the beginning of the investigation, agreement to conduct the research was obtained from the school dean.
Table 1 Characteristics of the study population of north Italian children, pre-adolescents and adolescents $(n 614), 2009$

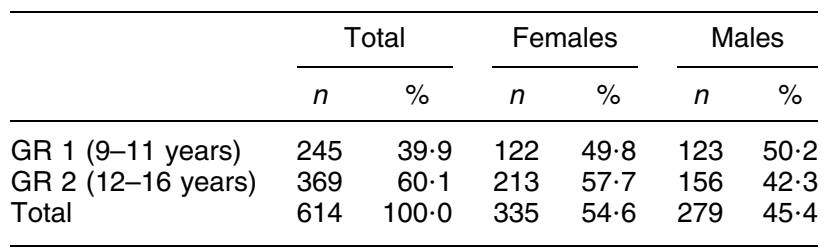

The exclusion criteria for the study were: (i) poor comprehension of the Italian language, i.e. the inability to read and learn the questions; (ii) age not included between the indicated ranges; and (iii) the questionnaires not being fully completed due to a lack of time.

The good compliance of the present study is evident from the small number of participants ${ }^{(9)}$ who were excluded: three students on the basis of the first criterion of exclusion, four students on the basis of the second one and two students on the basis of the third criterion. The rate of exclusion was $1.5 \%$. The total number of questionnaires on which the actual results were calculated is 614 .

The characteristics of the 614 participants are reported in Table 1.

\section{The QuesCA IT questionnaire}

The QuesCA IT questionnaire was filled in by the students in their classrooms in 2009. A section at the top of the questionnaire was to be completed with the age and gender of the participant.

The QuesCA IT questionnaire comprised thirty-one questions, only two of which were open questions (questions 8, 31); the other twenty-nine questions can be categorized as follows: (i) those with a true/false answer (questions 1-6, 9-17, 21, 22, 24-26, 28-30); (ii) multiplechoice questions with one correct answer (questions 7 , 18, 20); and (iii) multiple-choice questions with more than one correct answer question (questions 19, 23, 27). For each question the 'I don't know' answer was always present. The thirty-first question asked the students to evaluate their nutritional knowledge by tracing an X sign on a line. This line was successively divided into twenty major intervals corresponding to 10 values and then in other minor intervals corresponding to the half value. From the position of the $\mathrm{X}$ sign the corresponding value was thus calculated and the results obtained are presented in Table 6 . The answers were considered sufficient if greater than or equal to the value of 6 .

The thirty-one questions, all of the possible answers and the correct answer to each question are presented in Table 2. The questionnaires were completed anonymously, and evaluated for accuracy and completeness by the authors.

\section{Translation, adaptation and pre-test}

The questions in the QuesCA are clearly formulated and adopt a simple language, which allowed a simple translation. The Italian version, QuesCA IT, was submitted for 
Table 2 The condensed form of the nutritional knowledge questionnaire (QuesCA IT)

\begin{tabular}{ll}
\hline No. & Question \\
\hline 1. & Eating in a healthy way means to follow a restrictive diet \\
2. & Eating in a healthy way means to eat all kinds of food in \\
& a moderate way \\
3. & Breakfast promotes the consumption of snacks \\
4. & Breakfast helps you to concentrate \\
5. & A healthy snack allows you to vary your nutrition \\
6. & A healthy snack allows you not to arrive to the \\
7. & Choose the most balanced meal
\end{tabular}

8. Which beverage is to be preferred all over the day?

9. It is recommended to eat starchy foods at every meal

10. Starchy foods represent our main energy source

11. The consumption of starchy foods can increase weight

12. The consumption of starchy foods during the meal contributes to increase satiety

13. Cheese and meat contain the same quantity of protein

14. Eating meat/fish/eggs once or twice a day is healthy

15. Meat, fish and eggs help to build the muscular mass

16. It is recommended to eat fruit and vegetables regularly

17. It is recommended to eat fruit and vegetables regularly for their fibre content

18. How many portions of fruit and vegetables are recommended to eat a day?

19. Choose the foods which contain the most quantity of calcium (you can choose more than one answer)

20. At your age how many dairy products are recommended to eat?

21. Calcium is required to strengthen bones

22. Oils and butter also contain vitamins

23. Choose the foods which contain the most quantity of fats (you can choose more than one answer)

24. Eating fast foods once a month increases weight

25. Fast-food meals contain a lot of fats

26. In order to eat healthily fast foods are forbidden

27. Choose the foods which belong to the sweets group (you can choose more than one answer)

28. In a balanced diet it is possible to consume sweets

29. Sweets can be consumed once or twice a day

30. Beverages like Coca Cola contain little sugar

31. Try to evaluate your nutritional knowledge by drawing an $X$ on the line

Very bad

0
Possible answers

True; False; I don't know

True; False; I don't know

True; False; I don't know

True; False; I don't know

True; False; I don't know

True; False; I don't know

Pasta + green beans + strawberries; Chicken + green beans + pasta + a little oil + strawberries; Chicken + green beans + pasta + strawberries;

Chicken + green beans + a little oil + strawberries; I don't know

(open question)

True; False; I don't know

True; False; I don't know

True; False; I don't know

True; False; I don't know

True; False; I don't know

True; False; I don't know

True; False; I don't know

True; False; I don't know

True; False; I don't know

From 1 up to 3; From 7 up to 10 ; From 4 up to 6; I don't know

Cheese; Yoghurt, Butter; Ice cream; Cream; Milk; I don't know

From 1 up to 2; From 3 up to 4; From 2 up to 3 ; I don't know

True; False; I don't know

True; False; I don't know

Pop corn; Croissant; Peanuts; Puffed rice crackers/Milk bread; Packaged chips; I don't know

True; False; I don't know

True; False; I don't know

True; False; I don't know

Chocolate croissant; Natural yoghurt;

Candies; Sausage; Strawberry ice cream; I don't know

True; False; I don't know

True; False; I don't know

True; False; I don't know
Correct answer

False
True
False
True
True
True
Chicken + green
$\quad$ beans + pasta + a little
$\quad$ oil + strawberries

Water
True
True
False
True
True
True
True
True
True

From 4 up to 6

Cheese; Yoghurt; Milk

From 3 up to 4

True

True

Croissant; Peanuts;

Packaged chips

False

True

False

Chocolate croissant;

Candies; Strawberry

ice cream

True

True

False

Very good

10 consideration to a primary-school teacher to assess whether the language used was accessible to the same age group for which the QuesCA was designed and prepared. Later, a pre-test of the translated questionnaire was conducted among two bilingual Italian/French dietitians and a bilingual student in the last year of his study in Dietetics, in order to verify the correctness of the translation and its comprehensibility. Before the distribution of the questionnaire in the classrooms, a pilot experiment or a pre-test was performed within a group of ten young people aged 9-16 years which demonstrated the acceptance and feasibility of the survey. The questions of the Italian questionnaire and their order have been only marginally modified from QuesCA.
In particular: (i) the options 'rice' and 'salmon' (question 7 of the QuesCA) were replaced by 'pasta' and 'chicken' because the latter are most often consumed in Italy; and (ii) 'pain au chocolat' (question 27 of the QuesCA), a sweet like a croissant filled with chocolate and typically consumed in Switzerland, was replaced with 'chocolate croissant'. When present, the possibility of more than one correct answer was always indicated.

\section{Distribution of the questionnaire and collection procedure}

For the completion of the questionnaires the following rules were adopted: (i) experienced staff were present in 
the classroom from the distribution of questionnaires until their collection; (ii) neither the expert staff nor the teacher in the classroom could respond to any questions posed by the students; (iii) it was monitored that the students did not speak to each other and it was recommended to complete the questionnaire in all its parts, including the indication of age and gender; (iv) use of the 'I don't know' answer was clarified as the answer to choose in the absence of knowledge of the correct answer; (v) it was specified that the questionnaire was anonymous and would not affect the students' school marks; and (vi) students had the possibility to write questions or comments directly on the questionnaire. The time necessary to complete the questionnaire was evaluated as about $15-20 \mathrm{~min}$.

\section{Statistical analysis}

For the analysis of the answers the following procedure was adopted: a value of 1 was given to each correct answer, while to each incorrect or 'I don't know' answer a value of 0 was given. The questions were grouped on the basis of the different items of nutritional knowledge covered (Table 3) and then mean scores and standard deviations were calculated for each item and the whole questionnaire.

Table 3 The questions grouped on the basis of the different items covered

\begin{tabular}{lc}
\hline Item & Question no. \\
\hline Healthy diet & $1,2,7$ \\
Breakfast & 3,4 \\
Snacks & 5,6 \\
Fast food & $24,25,26$ \\
Beverages & 8,30 \\
Fruits and vegetables & $16,17,18$ \\
Cereals and tubers & $9,10,11,12$ \\
Meat/fish/legumes/eggs & $13,14,15$ \\
Milk and dairy food & $19,20,21$ \\
Fats and dressings & 22,23 \\
Sweets & $27,28,29$ \\
\hline
\end{tabular}

Gender and age group differences in mean scores for each item and the whole questionnaire were analysed by the two-sample Wilcoxon test (two-sided; Tables 4 and 5) using the statistical software package IBM SPSS Version $20 \cdot 0$. Age group differences in the self-assessment of nutritional knowledge (Table 6) were analysed by the $\chi^{2}$ test for non-parametric data using Microsoft ${ }^{\circledR}$ Excel 2003. $P<0.05$ was considered as statistically significant. To evaluate the effect size, Cohen's $d$ index for the standardized differences between two means was used ${ }^{(12)}$. This coefficient can be converted to the $r$ value, the effect size correlation, and vice versa.

\section{Results}

The data analysis highlighted that: (i) in GR1, $1.2 \%$ of students gave $<25 \%$ of correct answers, $98.4 \%$ of students gave $25-75 \%$ of correct answers and only $0.4 \%$ gave $>75 \%$ of correct answers, with scores in the range of 6-24 and corresponding mean score of $15 \cdot 8$ (sD 3.4, CI 0.4 ); (ii) in GR2, $0.5 \%$ of students gave $<25 \%$ of correct answers, $98 \cdot 1 \%$ of students gave $25-75 \%$ of correct answers and $1.4 \%$ gave $>75 \%$ of correct answers, with scores in the range of 4-23 and corresponding mean score of $16 \cdot 6$ (SD 2.9, CI $0 \cdot 3$ ). This difference was not statistically significant $\left(\chi^{2}\right.$ test, $P>0 \cdot 05$, data not shown).

The thirty-one questions of the QuesCA IT were grouped into eleven items (see Table 3 ) on the basis of their meaning and the concepts dealt with. This allowed data to be presented in a concise and clear way.

Considering the whole population, statistically significant gender differences were found for the items 'healthy diet', 'breakfast' and 'beverages', with the females' scores being higher than the males' scores. Among the younger students of GR1, the only statistically significant gender difference was related to the question item 'fast food', for which there

Table 4 Mean scores and standard deviations for the eleven items and the whole questionnaire according to gender and age group: north Italian children, pre-adolescents and adolescents ( $n 614), 2009$

\begin{tabular}{|c|c|c|c|c|c|c|c|c|c|c|c|c|}
\hline \multirow[b]{3}{*}{ Item (range of score) } & \multicolumn{4}{|c|}{ Total } & \multicolumn{4}{|c|}{ GR1 } & \multicolumn{4}{|c|}{ GR2 } \\
\hline & \multicolumn{2}{|c|}{ Females } & \multicolumn{2}{|c|}{ Males } & \multicolumn{2}{|c|}{ Females } & \multicolumn{2}{|c|}{ Males } & \multicolumn{2}{|c|}{ Females } & \multicolumn{2}{|c|}{ Males } \\
\hline & Mean & SD & Mean & SD & Mean & SD & Mean & SD & Mean & SD & Mean & SD \\
\hline Healthy diet (0-3) & $1 \cdot 73^{\star *}$ & 0.79 & $1 \cdot 54$ & 0.81 & $1 \cdot 62$ & 0.68 & 1.54 & $0 \cdot 80$ & $1 \cdot 79^{\star *}$ & 0.83 & 1.54 & $0 \cdot 81$ \\
\hline Breakfast (0-2) & $1 \cdot 54^{\star \star}$ & $0 \cdot 60$ & $1 \cdot 32$ & 0.72 & $1 \cdot 39$ & 0.64 & $1 \cdot 26$ & 0.74 & $1 \cdot 63^{\star \star}$ & 0.56 & $1 \cdot 37$ & 0.69 \\
\hline Snacks $(0-2)$ & $1 \cdot 13$ & 0.59 & $1 \cdot 11$ & 0.67 & $1 \cdot 14$ & 0.57 & $1 \cdot 14$ & 0.69 & $1 \cdot 12$ & 0.60 & 1.09 & 0.66 \\
\hline Fast food $(0-3)$ & $2 \cdot 14$ & $0 \cdot 84$ & $2 \cdot 20$ & 0.75 & $1 \cdot 81^{\star *}$ & 0.88 & $2 \cdot 11$ & $0 \cdot 76$ & $2 \cdot 32$ & 0.75 & $2 \cdot 28$ & 0.74 \\
\hline Beverages (0-2) & $1 \cdot 80^{\star *}$ & 0.45 & $1 \cdot 64$ & 0.57 & $1 \cdot 75$ & 0.49 & $1 \cdot 63$ & 0.63 & $1 \cdot 83^{\star *}$ & 0.42 & $1 \cdot 65$ & 0.52 \\
\hline Fruits and vegetables $(0-3)$ & 1.98 & 0.72 & $1 \cdot 90$ & 0.77 & 1.97 & 0.72 & $1 \cdot 89$ & $0 \cdot 70$ & 1.99 & 0.72 & 1.91 & 0.82 \\
\hline Cereals and tubers $(0-4)$ & $2 \cdot 00$ & $1 \cdot 03$ & 1.96 & $1 \cdot 06$ & 1.90 & $1 \cdot 03$ & $1 \cdot 87$ & $1 \cdot 08$ & $2 \cdot 05$ & $1 \cdot 03$ & $2 \cdot 03$ & $1 \cdot 05$ \\
\hline Meat/fish/legumes/eggs (0-3) & $1 \cdot 17$ & 0.76 & $1 \cdot 26$ & 0.80 & $1 \cdot 28$ & 0.78 & $1 \cdot 32$ & 0.80 & $1 \cdot 11$ & 0.74 & $1 \cdot 21$ & 0.80 \\
\hline Milk and diary food (0-3) & $1 \cdot 44$ & 0.62 & 1.39 & 0.66 & $1 \cdot 41$ & 0.68 & $1 \cdot 39$ & 0.69 & $1 \cdot 46$ & 0.59 & $1 \cdot 40$ & 0.64 \\
\hline Fats and dressings (0-2) & $0 \cdot 21$ & 0.43 & $0 \cdot 19$ & $0 \cdot 41$ & $0 \cdot 16$ & $0 \cdot 41$ & $0 \cdot 18$ & $0 \cdot 41$ & 0.23 & 0.44 & $0 \cdot 21$ & 0.42 \\
\hline Sweets $(0-3)$ & $1 \cdot 41$ & 0.78 & $1 \cdot 49$ & 0.81 & $1 \cdot 40$ & 0.72 & $1 \cdot 44$ & 0.80 & $1 \cdot 41$ & 0.81 & $1 \cdot 53$ & 0.82 \\
\hline Whole questionnaire $(0-30)$ & $16 \cdot 54$ & $2 \cdot 90$ & $16 \cdot 02$ & $3 \cdot 40$ & $15 \cdot 84$ & 3.34 & $15 \cdot 76$ & 3.44 & $16 \cdot 94$ & $2 \cdot 54$ & $16 \cdot 22$ & 3.36 \\
\hline
\end{tabular}

GR1, 9-11 years; GR2, 12-16 years.

Mean values were significantly different from those of males (considered in the total sample and separately by age group): ${ }^{* \star} P<0 \cdot 01$. 
Table 5 Comparison of mean scores and standard deviations of the eleven questionnaire items in females and males according to age group: north Italian children, pre-adolescents and adolescents ( $n$ 614), 2009

\begin{tabular}{|c|c|c|c|c|c|c|c|c|}
\hline \multirow[b]{3}{*}{ Item (range of score) } & \multicolumn{4}{|c|}{ Females } & \multicolumn{4}{|c|}{ Males } \\
\hline & \multicolumn{2}{|c|}{ GR1 } & \multicolumn{2}{|c|}{ GR2 } & \multicolumn{2}{|c|}{ GR1 } & \multicolumn{2}{|c|}{ GR2 } \\
\hline & Mean & SD & Mean & SD & Mean & SD & Mean & SD \\
\hline Healthy diet (0-3) & $1 \cdot 62$ & 0.68 & $1 \cdot 79$ & 0.83 & $1 \cdot 54$ & $0 \cdot 80$ & 1.54 & $0 \cdot 81$ \\
\hline Breakfast $(0-2)$ & $1 \cdot 39$ & 0.64 & $1 \cdot 63^{\star *}$ & 0.56 & $1 \cdot 26$ & $0 \cdot 74$ & $1 \cdot 37$ & 0.69 \\
\hline Snacks $(0-2)$ & $1 \cdot 14$ & 0.57 & $1 \cdot 12$ & 0.60 & $1 \cdot 14$ & 0.69 & 1.09 & 0.66 \\
\hline Fast food $(0-3)$ & $1 \cdot 81$ & 0.88 & $2 \cdot 32^{\star *}$ & 0.75 & $2 \cdot 11$ & $0 \cdot 76$ & $2 \cdot 28$ & $0 \cdot 74$ \\
\hline Beverages $(0-2)$ & $1 \cdot 75$ & 0.49 & $1 \cdot 83$ & 0.42 & $1 \cdot 63$ & 0.63 & $1 \cdot 65$ & 0.52 \\
\hline Fruits and vegetables $(0-3)$ & 1.97 & 0.72 & 1.99 & 0.72 & $1 \cdot 89$ & $0 \cdot 70$ & 1.91 & 0.82 \\
\hline Cereals and tubers $(0-4)$ & 1.90 & 1.03 & 2.05 & 1.03 & $1 \cdot 87$ & $1 \cdot 08$ & $2 \cdot 03$ & 1.05 \\
\hline Meat/fish/legumes/eggs (0-3) & $1 \cdot 28$ & 0.78 & $1 \cdot 11^{*}$ & 0.74 & $1 \cdot 32$ & $0 \cdot 80$ & $1 \cdot 21$ & 0.80 \\
\hline Milk and dairy food $(0-3)$ & $1 \cdot 41$ & 0.68 & 1.46 & 0.59 & $1 \cdot 39$ & $0 \cdot 69$ & $1 \cdot 40$ & 0.64 \\
\hline Fats and dressings $(0-2)$ & $0 \cdot 16$ & 0.41 & 0.23 & $0 \cdot 44$ & $0 \cdot 18$ & 0.41 & $0 \cdot 21$ & 0.42 \\
\hline Sweets $(0-3)$ & 1.40 & 0.72 & $1 \cdot 41$ & 0.81 & $1 \cdot 44$ & $0 \cdot 80$ & 1.53 & 0.82 \\
\hline
\end{tabular}

GR1, 9-11 years; GR2, 12-16 years.

Mean values were significantly different from those of the younger children: ${ }^{\star} P<0 \cdot 05,{ }^{\star \star} P<0 \cdot 01$.

Table 6 Answers to question 31 (self-assessment of nutritional knowledge) according to age group: north Italian children, preadolescents and adolescents ( $n$ 614), 2009

\begin{tabular}{lrc}
\hline & GR1 & GR2 \\
\hline Insufficient or negative assessment & & \\
$n$ & 28 & 89 \\
$\%$ & 11 & $24^{* *}$ \\
Sufficient or positive assessment & 217 & 280 \\
$n$ & 89 & $76^{* *}$ \\
$\%$ & &
\end{tabular}

GR1, 9-11 years; GR2, 12-16 years.

Proportions were significantly different from those of the younger children $\left(\chi^{2}\right.$ test $):{ }^{\star \star} P<0 \cdot 01$.

was a lower score in the female group. In the case of the older students in GR2, statistically significant gender differences were seen in relation to the items 'healthy diet', 'breakfast' and 'beverages'. In all these cases, the mean scores were always higher in females than in males (all $P<0 \cdot 01$; see Table 4). Comparison between the answers given by females according to age group revealed that: (i) for question items 'breakfast' and 'fast food', GR2 had statistically significantly higher mean scores than GR1 $(P<0 \cdot 01)$; and (ii) for the question item 'meat/fish/ legumes/eggs', the mean score of GR1 was statistically significantly higher than that of GR2 $(P<0.05)$. On the contrary, the comparison between answers given by males according to age group evidenced no statistically significant differences (Table 5).

The answers to the final question are presented in Table 6. Eleven per cent of the GR1 population judged its nutritional knowledge insufficient or negative $v .24 \%$ in the GR2 population ( $\chi^{2}$ test, $P<0 \cdot 01$ ); accordingly the majority of GR1 (89\%) was more positive about its nutritional knowledge compared with GR2 (76\%); $\chi^{2}$ test, $P<0 \cdot 01)$.

The effect size analysis revealed that the percentage of non-overlap was: (i) $0 \cdot 0 \quad(r=0 \cdot 0$, Cohen's $d=0 \cdot 006)$ between males and females belonging to GR1; (ii) $21 \cdot 3$ ( $r=0 \cdot 3$, Cohen's $d=0 \cdot 708)$ between males and females belonging to GR2; (iii) $33 \cdot 0(r=0 \cdot 5$, Cohen's $d=1 \cdot 153)$ between females of the two age groups; and (iv) $21 \cdot 3$ $(r=0 \cdot 3$, Cohen's $d=0.556$ ) between males of the two age groups.

\section{Discussion}

The present work was undertaken to explore the real nutritional knowledge of an Italian population of children, pre-adolescents and adolescents through the completion of a validated questionnaire. The current investigation is different from other similar surveys since the investigated population is larger, including children (9-11 years old) and adolescents (12-16 years old), and because it takes account not only particular issues in nutrition science, such as the consumption of fruits and vegetables $^{(13)}$ and eating behaviours ${ }^{(14)}$ of for instance soft drinks and junk foods, but also how to choose the more balanced meal among many.

The importance of knowing what a 'healthy diet' means arises also from the need for young people to understand when nutrition advertisements in the media are safe or unhealthy.

From data obtained and presented herein, some considerations can be made. First of all, the item that accounts for the lowest score both in males and females is 'fats and dressings', followed by the items 'snacks', 'meat/ fish/legumes/eggs', 'milk and dairy foods' and 'sweets'. That the students had low scores for 'fats and dressings' and 'meat/fish/legumes/eggs' provides evidence that the exact role of the macronutrients protein and fat in a healthy diet is far from known and a lot of work must be done to improve this result. The item 'snacks' represents a hot issue for adolescents, since a part of them believes 
consuming snacks is wrong while the other part consumes junk food as snacks.

In the case of the 'healthy diet' item, the statistically significant differences found are probably due to the presence of a complex question (question 7) with a multiple-choice answer, since it assumes that the students have exact knowledge on all the nutrients and how to combine them for a balanced diet. Analogously, the items 'breakfast', 'fast food' and 'beverages', for which there are statistically significant differences between males and females, comprise questions about a healthy lifestyle and thus a healthy diet.

The mean scores as a whole were higher, although not statistically significantly so, for GR2 compared with GR1; a result showing that underlying nutritional knowledge, but possibly more general knowledge, increases with age. This is in agreement with results obtained by the HELENA (Healthy Lifestyle in Europe by Nutrition in Adolescence) study ${ }^{(15)}$ and by the parallel study performed using the original Swiss QuesCA questionnaire ${ }^{(11)}$; thus it seems a general observation in the European child and adolescent population. Moreover, in the present study the answers by females almost always reached a higher value of correctness, a result again in agreement with the HELENA study ${ }^{(15)}$. This is not surprising since it is a common opinion that girls have more interest in and care for their health and physical appearance than boys, a result that also confirms pre-existing studies ${ }^{(13-19)}$. This specific finding could be considered in order to plan interventions in the case of educational projects, so as to find more appealing forms to involve also boys. All of these differences were confirmed by the effect size analysis as moderately effective; thus the different sample sizes of the populations examined (GR1 $v$. GR2, females $v$. males) only marginally influenced the results obtained.

A last comment can be drawn from the self-assessment of nutritional knowledge, since the higher percentage of insufficient level in the GR2 population can be regarded as a higher degree of self-consciousness and of the lack of important information, another parameter to consider in planning an educational project.

A limitation of the present study can be found in the lack of any association between our data and other parameters such as information on pre-existing knowledge in nutrition coming from the school or the family, on anthropometric measures such as weight, height and level of physical activity, and without any correlation with socio-economic indices such as parents' educational level and financial status; but it is due to a precise decision in our project. The lack of any planned nutritional education moment in Italian schools, except for some voluntary projects, has led to a self-informed young population, to this population - irrespective of their socio-economic or other information - and will have directed the future action of intervention in their heterogeneous classrooms. All of these data were collected before the Italian Government decided to introduce nutritional education as a teaching subject in primary and secondary schools, but in the same period of the HELENA study which is more distributed across Italy. The teaching of nutritional education could be the first and main action to do, since students' knowledge on macronutrients and their role is low. The nutritional education should be performed in more than one way, not only during school time, but also at supermarkets for instance, in order to obtain a massive campaign of information.

The second action could be to find educational forms not too aggressive or boring, trying to explain the nutrition labelling on food packages for example, in order to make students aware of their choices. It would be more appropriate to try to use the language and the 'way of life' of the population in the survey. The results obtained herein, although relative to the two north Italian towns of Milan and Bergamo, and very similar to the ones obtained by the HELENA study, support the choice of the Italian Government to introduce nutritional education as a teaching subject and provide a base from which to plan interventions directed to young people.

\section{Acknowledgements}

Source of funding: This research received no specific grant from any funding agency in the public, commercial or not-for-profit sectors. Conflicts of interest: There are no conflicts of interest to declare. Ethics: Ethical approval was not required. Authors' contributions: A.T. did all the work in the schools, prepared and filled the database with the students' answers, helped compile the tables and contributed to writing the paper. A.Z. conducted the statistical analysis and compiled the tables. A.F. analysed the data, advised on data representation and wrote the paper. Acknowledgements: The authors thank Professor M. Kruseman for her teachings and above all for the possibility she gave to A.T. to join the QuesCA work group; Professor A.E. Pontiroli for his help in organizing the Italian work; J. Truan, L. Duboule and the Haute École de Santé de Genève, Filière Nutrition et Diététique, for collaboration at various levels; and the Italian schools for agreeing to participate to the survey.

\section{References}

1. Wardle J, Parmenter K \& Walzer J (2000) Nutrition knowledge and food intake. Appetite 34, 269-275.

2. Johnson F, Wardle J \& Griffith J (2002) The adolescent food habits checklist: reliability and validity of a measure of healthy eating behaviour in adolescents. Eur J Clin Nutr 56, 644-649.

3. Stafleu A, Van Staveren WA, De Graaf C et al. (1996) Nutrition knowledge and attitudes towards high-fat foods and low-fat alternatives in three generations of women. Eur J Clin Nutr 50, 33-41.

4. Contento IR, Randell JS \& Basch CE (2002) Review and analysis of evaluation measures used in nutrition education intervention research. J Nutr Educ Behav 34, 2-25. 
5. Anderson AS, Umapathy D, Palumbo L et al. (1988) Nutrition knowledge assessed in a group of medical inpatients. J Hum Nutr Diet 1, 39-46.

6. McDougall P (1998) Teenagers and nutrition: assessing levels of knowledge. Health Visitor 71, 62-64.

7. Shepherd R \& Stockley L (1987) Nutrition knowledge, attitudes and fat consumption. I Am Diet Assoc 87, 615-619.

8. McDonnell GE, Roberts DCK \& Lee C (1998) Stages of change and reduction of dietary fat: effect of knowledge and attitudes in an Australian university population. $J$ Nutr Educ 30, 37-44.

9. Levy AS, Fein SB \& Stephenson M (1993) Nutrition knowledge levels about dietary fat and cholesterol. J Nutr Educ 25, 60-66.

10. Steenhuis IHM, Brug J, Van Assema P et al. (1996) The validation of a test to measure knowledge about the fat content of food products. Nutr Health 10, 331-339.

11. Kruseman M, Berchtold A, Truan J et al. (2012) Développement et validation du QuesCA, un Questionnaire permettant d'évaluer les Connaissances Alimentaires auprès de jeunes de 9 à 15 ans. Arch Pediatr 19, 456-466.

12. Cohen J (1988) Statistical Power Analysis for the Behavioral Sciences, 2nd ed. Hillsdale, NJ: Lawrence Erlbaum.
13. Sandvik C, De Bourdeaudhuij I, Due P et al. (2005) Personal, social and environmental factors regarding fruit and vegetable intake among schoolchildren in nine European countries. Ann Nutr Metab 49, 255-266.

14. Triches RM \& Giugliani RJ (2005) Obesity, eating habits and nutritional knowledge among school children. Rev Saude Publica 39, 541-547.

15. Sichert-Hellert W, Beghin L, De Henauw S et al. (2011) Nutritional knowledge in European adolescents: results from the HELENA (Healthy Lifestyle in Europe by Nutrition in Adolescence) study. Public Health Nutr 14, 2083-2091.

16. Osler M \& Hansen ET (1993) Dietary knowledge and behavior among schoolchildren in Copenhagen, Denmark. Scand J Soc Med 21, 135-140.

17. Beech BM, Rice R, Myers I et al. (1999) Knowledge attitudes and practices related to fruit and vegetable consumption of high school students. J Adolesc Health 24, 244-250.

18. Pirouznia M (2001) The association between nutrition knowledge and eating behavior in male and female adolescents in the US. Int J Food Sci Nutr 52, 127-132.

19. Godina-Zarfel B \& Elmadfa I (1993) Food preferences, nutritional knowledge and their impact on nutrient intake in Austrian children and adolescents. Nutrition 17, 314-315. 